\title{
Electrospray Ionization Mass Spectrometry of Biotin Binding to Streptavidin
}

\author{
Klaus Eckart and Joachim Spiess \\ Department of Molecular Neuroendocrinology, Max Planck Institute for Experimental Medicine, \\ Goettingen, Germany
}

\begin{abstract}
Stepwise binding of biotin to streptavidin via several intermediates was monitored with electrospray ionization mass spectrometry (ESIMS). Protein ligand interactions that result in conformational changes could be recognized with ESIMS by a mass shift and a change of the average multiple charge state of this protein. In addition, mass spectrometry for the ions in the gas phase revealed a much greater strength of the noncovalent bonds between the streptavidin subunits in the tetrameric complex than between the streptavidin and biotin molecules and remarkable differences in stability for the different charge states of the biotin-streptavidin noncovalent complex. (J Am Soc Mass Spectrom 1995, 6, 912-919)
\end{abstract}

C hanges of protein conformation during interaction with specific ligands [1] are of great importance to understand function. Investigations can be carried out with x-ray crystallography or nuclear magnetic resonance (NMR). Especially x-ray crystallography can provide detailed structural information even on large protein complexes such as yeast hexokinase [2]. Such information cannot be obtained with NMR because of the size of these proteins.

Recently the unfolding and folding processes of myoglobin were demonstrated with electrospray ionization mass spectrometry (ESIMS) $[3,4]$. The unfolding of myoglobin induced by acidification was shown to result in a dramatic shift of the distribution pattern of multiply charged myoglobin forms. Native myoglobin carries an average of 9 protons per molecule, whereas an average of approximately $15-20$ protons is attached to unfolded myoglobin. This change reflects the size of the protein surface and does not necessarily depend on the number of basic sites of the protein surface. This particular example showed that the number of basic sites on the protein surface was always much greater than the number of protons [4].

The adaptation of ESIMS for aqueous solutions [5] enabled the investigation of noncovalent protein ligand or protein substrate complexes $[3,6,7]$. The detection of protein subunit complexes also recently was described for different proteins [8-19], and the possibilities and limitations for the observation of noncovalent interactions with ESIMS were addressed [20]. It was stated that the observation of noncovalent complexes did not necessarily imply the specificity of this interaction. One important criterion for specific interac-

Address reprint requests to Klaus Eckart, Department of Molecular Neuroendocrinology, Max ['lanck Institute for Experimental Medicine. Hermann Rein Strasse 3, D 37075 Gixettingen, Cermany tions can be the exclusive presence of the complex in the electrospray ionization (ESI) mass spectrum.

More recently, noncovalent complexes of proteins that include the avidin tetramer were investigated with a low frequency extended mass range quadrupole electrospray mass spectrometer [12]. It was demonstrated by average mass shift that four molecules of biotin were bound to avidin [21]. In addition, the most prominent charged state of $+16(Q 16)$ for tetrameric avidin was change to +15 (Q15) for tetrameric avidin after binding four biotin molecules. This difference was assumed to reflect the different constraints in the noncovalent complex. Unfortunately, due to the low instrumental resolution of this particular type of quadrupole mass spectrometer, no identification of different biotinylation states was possible. An analysis of the differently biotinylated tetrameric streptavidin noncovalent complex can be of great importance for observation of the equilibrium at different ligand concentrations with an excess of streptavidin.

We report here for the first time sector field mass spectrometric results that indicate the progress of the binding process during stepwise ligand addition by the separation and identification of partially biotinylated streptavidin tetrameric complexes, which represent the reaction intermediates of the biotin saturation process. In addition, the dissociation of the noncova lent complex into smaller components in the interface by collisional-activated decompositions (CAD) provides some insight into the relative gas-phase stability of the different noncovalent bonds in the complex.

Investigation of conformational changes generally may be used to study ligand binding to proteins with ESIMS. Due to the induced fit, a specific noncovalent ligand protein interaction should induce a significant change in the conformational constraints of the protein, which is expected to be accompanied by a change of 
the average number of charges observed in the ESI mass spectrum. This effect already was reported for biotin binding to avidin [21] and also appears to take place with biotin binding to streptavidin, as described in subsequent text. This method is currently being developed in this laboratory to analyze the activation or modulation of receptors controlled by binding of specific ligands.

Streptavidin produced by Streptomyces avidinii was chosen because it is not glycosylated. Thus, the possibilities for microheterogeneity are reduced and highly resolved signals are provided in ESIMS. The binding properties of avidin and streptavidin toward biotin are known to be equivalent [22], but usually avidin is used because of its much lower price [23].

\section{Experimental}

Mass spectra were recorded on a Fisons (Manchester, UK) VG AutoSpec-T tandem mass spectrometer equipped with an array detector and an electrospray interface. This interface (Figure 1) contained a spray chamber with a pepperpot counter electrode, a stainless steel spray needle for better stability (especially when spraying from water solutions), and a hexapole for higher ion transmission through the interface. The instrument control and data evaluation were performed with the OPUS V3.1 data system running on a DEC Station 3000/300 LX (Digital Equipment Corp., Maynard, CA).

Samples were applied to the electrospray interface with a loop injection system. The mobile phase was delivered via an infusion syringe pump (Model 22, Harvard Apparatus, South Natick, MA) with a flow rate of $5 \mu \mathrm{L} / \mathrm{min}$. Samples were injected into a $20-\mu \mathrm{L}$ stainless steel loop of a Rheodyne (Cotati, CA) 8125 injector. The injector was connected with the spray needle through a $1-\mathrm{m}$ fused silica capillary of $50-\mu \mathrm{m}$ inside diameter. The spray chamber was heated constantly to $80{ }^{\circ} \mathrm{C}$ when methanol-containing solutions were being sprayed and was not heated for the investigation of streptavidin biotin binding in $10-\mathrm{mM}$ ammonium acetate. The spray was stabilized with a coaxial nitrogen stream of $14 \mathrm{~L} / \mathrm{h}$ for methanol-containing solutions and an oxygen stream of $14 \mathrm{~L} / \mathrm{h}$ for solutions free of methanol. Desolvation was supported by nitrogen flow $(300 \mathrm{~L} / \mathrm{h})$ through the spray chamber. The interface voltages were as follows (Figure 1): needle voltage, $8000 \mathrm{~V}$; counterelectrode, $5000 \mathrm{~V}$; sampling cone voltage, between 4020 and $4150 \mathrm{~V}$ as indicated in the figures; acceleration voltage or skimmer voltage, $4000 \mathrm{~V}$; ring electrode, $40 \mathrm{~V}$ below skimmer voltage.

For calibration a saturated aqueous CsI solution diluted 1:50 with a mixture of $49.5 \%$ water, $49.5 \%$ methanol, and $1 \%$ acetic acid was employed. Spectra were recorded from $m / z$ 8000-500 or $\mathrm{m} / \mathrm{z} 6000-1000$ by exponential down scans with a scan speed of $5 \mathrm{~s}$ per decade. The instrument was set to 1000 mass resolution ( $10 \%$ valley definition). Streptavidin (Boehringer Mannheim, Mannheim, Germany) was dissolved at a concentration of $10 \mu \mathrm{g} / \mu \mathrm{L}$ in water. The D-biotin binding capacity was given as $>14-\mu \mathrm{g}$ D-biotin per milligram of streptavidin. This leads to a molarity of greater than $140 \mu \mathrm{M}$ for the streptavidin tetramer. D-biotin (Boehringer Mannheim) was dissolved to a final concentration of $0.25(1 \mathrm{mM})$ and $0.05 \mu \mathrm{g} / \mu \mathrm{L}$ $(200 \mu \mathrm{M})$ in $10-\mathrm{mM}$ ammonium acetate $(\mathrm{pH} 8)$. For ESIMS the streptavidin stock solution was diluted 1:10 in 10- $\mathrm{mM}$ ammonium acetate $(\mathrm{pH} 8)$ to a final concentration of $1 \mu \mathrm{g} / \mu \mathrm{L}$. Appropriate amounts of the biotin stock solutions were added for the binding experiments. The final solutions were freshly prepared shortly before the measurements. No changes were observed after a few hours incubation at room temperature.

\section{Results and Discussion}

Determination of the streptavidin molecular mass resulted in a 3.5-ku difference compared with the calculation from the database sequence. Earlier observations
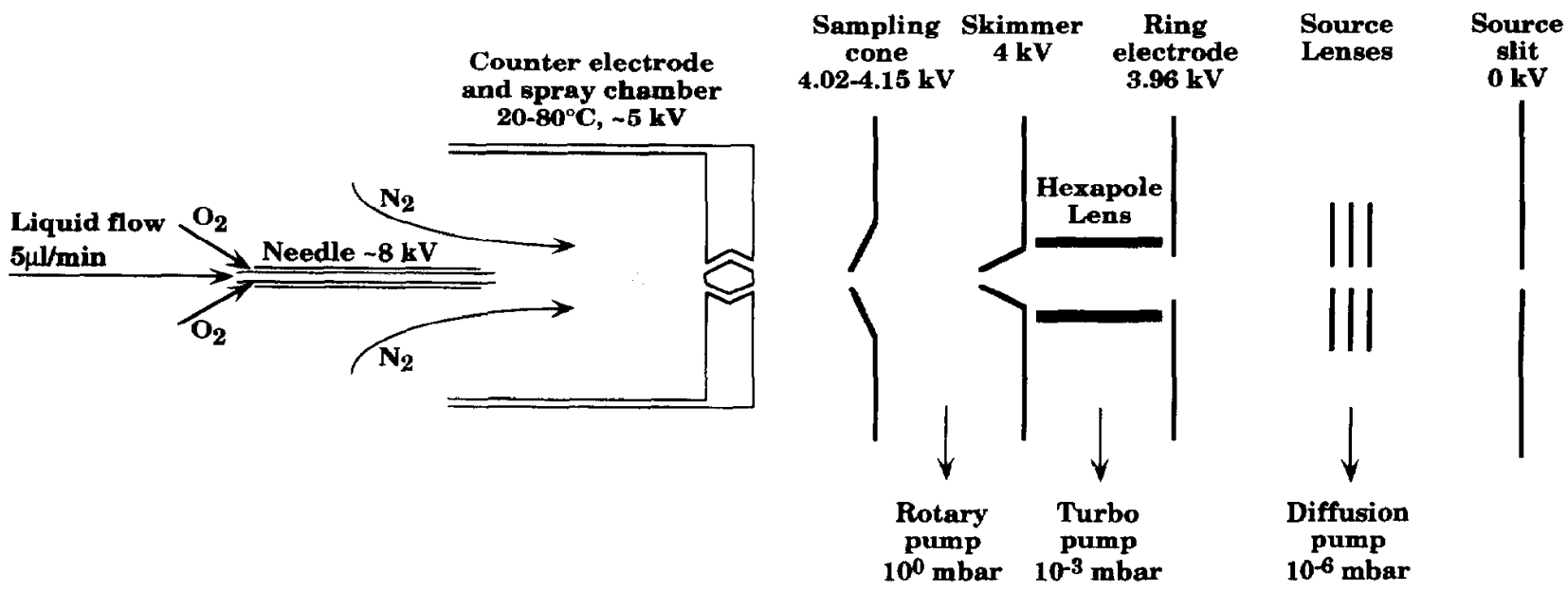

Figure 1. Schematic representation of the Fisons design electrospray interface. 
indicated that streptavidin also may be produced in smaller sizes as a result of enzymatic degradation during fermentation [22]. In fact, the streptavidin used here (Boehringer Mannheim) represents the so-called core streptavidin cleaved at the C-terminal side of Ala (13) and Ser (136). Its molecular mass was measured to be 12,970.9 (calculated 12,971.1). The sequence was validated by Edman degradation of the $20 \mathrm{~N}$-terminal amino acid residues. These data are in agreement with earlier findings [24].

On the basis of experience obtained with ESIMS on noncovalent complexes [20] the conditions of the electrospray interface were optimized. It was decided to minimize excitation of the molecules during electrospray and transport through the interface. This objective was achieved with an electrospray at room temperature and a relatively low sampling cone voltage offset of $40 \mathrm{~V}$. Under these conditions negligible amounts $(<1 \%)$ of streptavidin trimer and monomer were observed (Figure 2a), and three weak and broad peaks that represent the streptavidin octamer were detected between $m / z 4300$ and 4800 . Increase of the cone voltage offset to $90 \mathrm{~V}$ changed the spectrum significantly (Figure 2b). As expected the streptavidin tetramer started to dissociate to a significant extent mainly into the trimer and the monomer species as described earlier for avidin [21]. The formation of the smaller streptavidin oligomers with increasing sam- pling cone voltage was in agreement with the expectation that streptavidin is exclusively present as the tetramer in solution. In addition, the more efficient declustering provided higher resolution of the tetramer ions, but as we subsequently show, even under conditions without tetramer dissociation separation of the different biotinylation products was achieved.

\section{Biotin Binding Experiments}

The binding experiments were carried out with a streptavidin concentration of $1 \mu \mathrm{g} / \mu \mathrm{L}$. Theoretically, $56-76-\mu \mathrm{M}$ biotin is needed for saturation of the tetrameric streptavidin with biotin. This range is calculated from the minimum biotin binding capacity of streptavidin given by the supplier (see also Experimental) and the weight of the protein. Experimentally, saturation was observed between 60 and $80 \mu \mathrm{M}$ (Figure 3). Above $80 \mu \mathrm{M}$, no significant changes in the mass spectral patterns could be detected. The spectrum of $40-\mu \mathrm{M}$ biotin (Figure $3 \mathrm{c}$ ) showed slightly more than $50 \%$ saturation of streptavidin with biotin. These experimental results matched the previously mentioned theoretical range for the binding capacity of streptavidin. Even under conditions of biotin saturation, loss of biotin from the 115 charged (Q15) strep tavidin tetramer was observed, whereas the loss of biotin from + 16 charged (Q16) was negligible (Figure

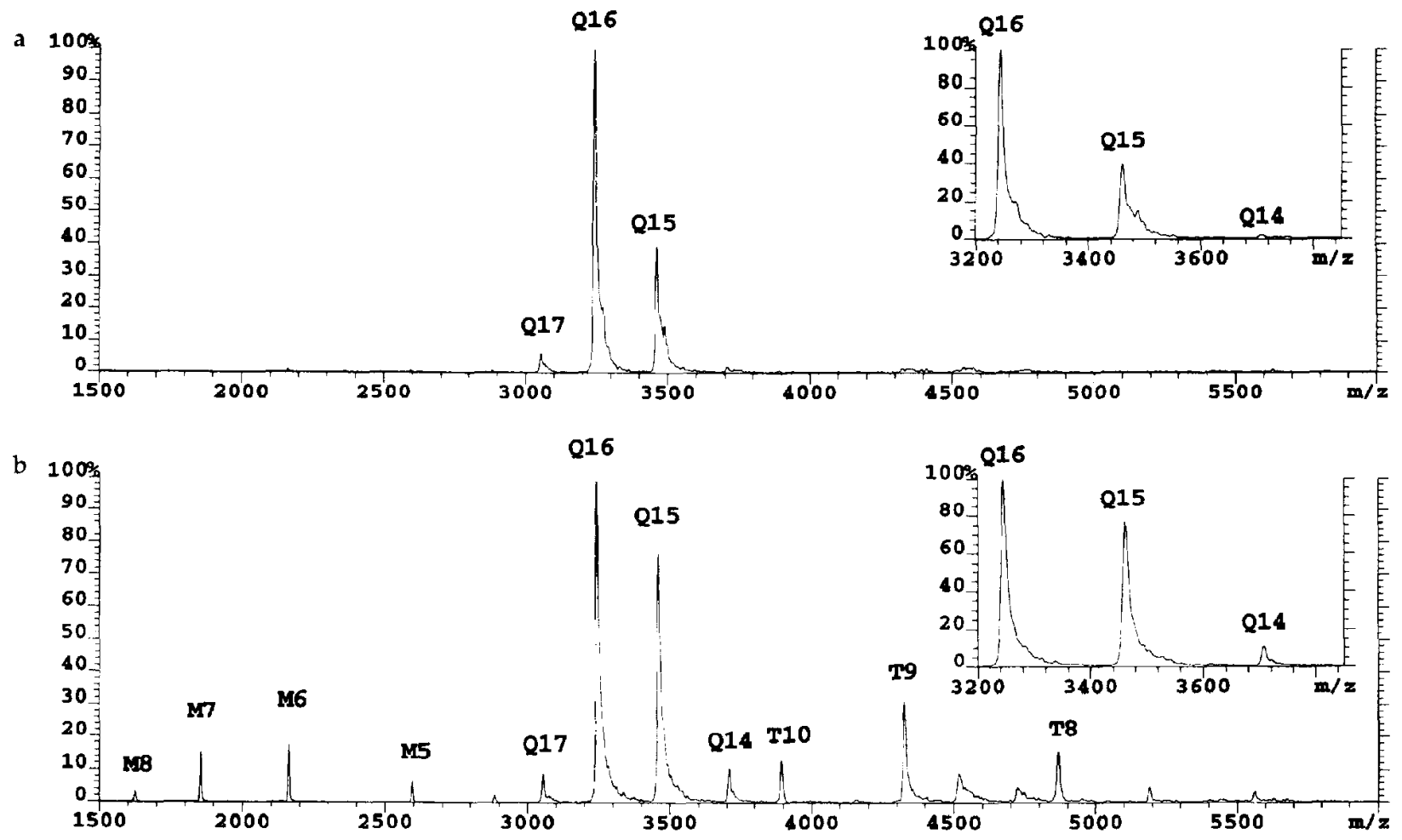

Figure 2. Partial ESI mass spectra of $1-\mu \mathrm{g} / \mu \mathrm{L}$ streptavidin in $10-\mathrm{mM}$ ammonium acetate buffer, $\mathrm{pH} 8$, recorded at cone voltage offsets of (a) $40 \mathrm{~V}$ and (b) $90 \mathrm{~V}$. The insets show the expanded mass region of the tetramer. Labels: $M$, monomer; $T$, trimer; $Q$, tetramer; the number gives the number of protons. 


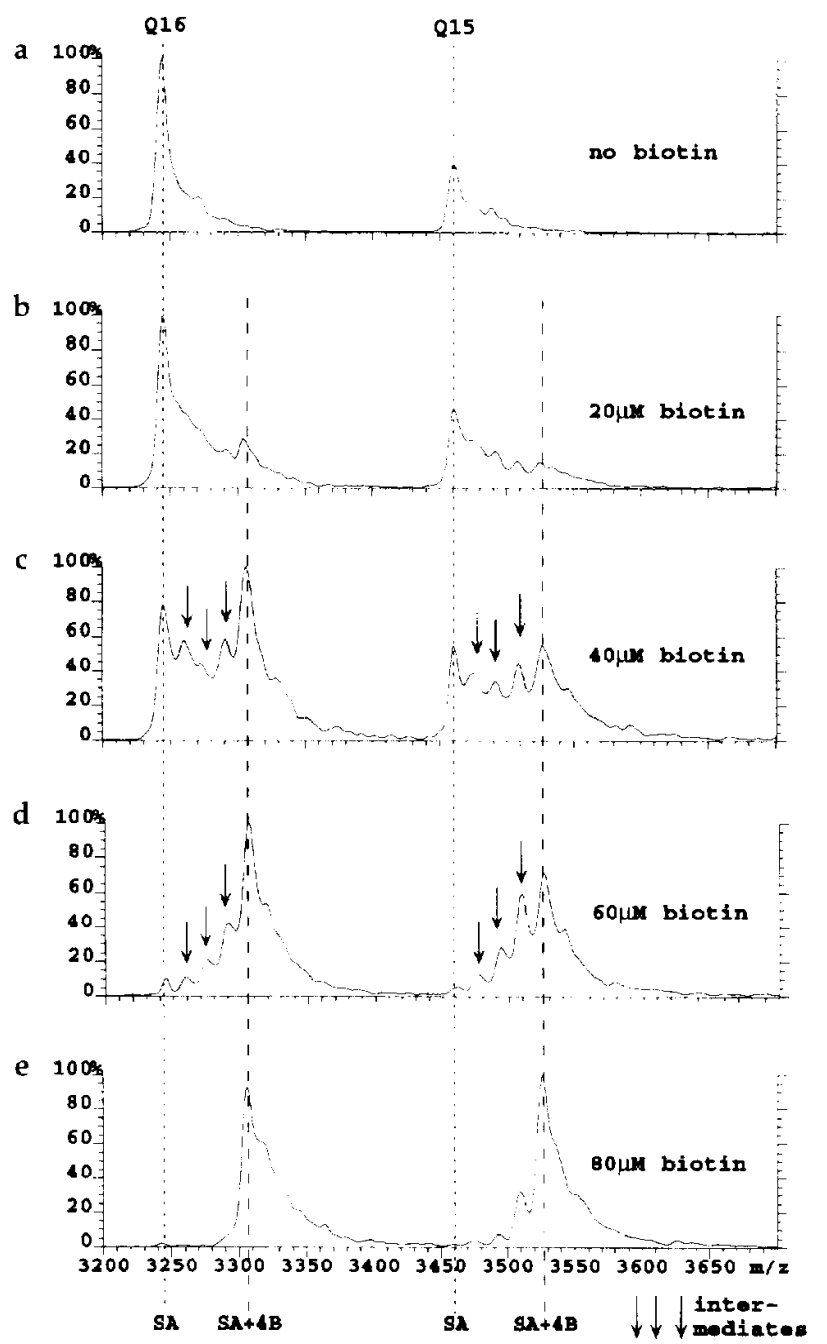

Figure 3. Partial ESI mass spectra of $1-\mu \mathrm{g} / \mu \mathrm{L}$ streptavidin in 10-mM ammonium acetate buffer, $\mathrm{pH} 8$, with different concentrations of biotin recorded at a cone voltage of fset of $40 \mathrm{~V}$ : (a) No biotin; (b) $20-\mu \mathrm{M}$ biotin; (c) $40-\mu \mathrm{M}$ biotin; (d) $60-\mu \mathrm{M}$ biotin; (e) $80-\mu \mathrm{M}$ biotin. Labels: $\mathrm{Q}$, tetramer; the number gives the number of protons; SA, streptavidin; $4 \mathrm{~B}$, four molecules biotin.

3e). As a result of these observations it was decided to use peak areas instead of peak heights as a measure of the abundance for the different charge states of nonbiotinylated and biotinylated tetrameric streptavidin.

The charge state pattern of fully biotinylated streptavidin was found to be significantly different from nonbiotinylated streptavidin (Figure 3) as described earlier for avidin [21]. In view of the above-mentioned decompositions of the streptavidin biotin noncovalent complex, application of relatively mild declustering conditions was necessary. In addition, for a clearer representation of the change in the charge state pattern, the signals for Q15 and Q16 with and without biotin were integrated (Figure 4). These data demonstrated the conformational change induced by specific biotin binding.

The experimentally determined mass values for the

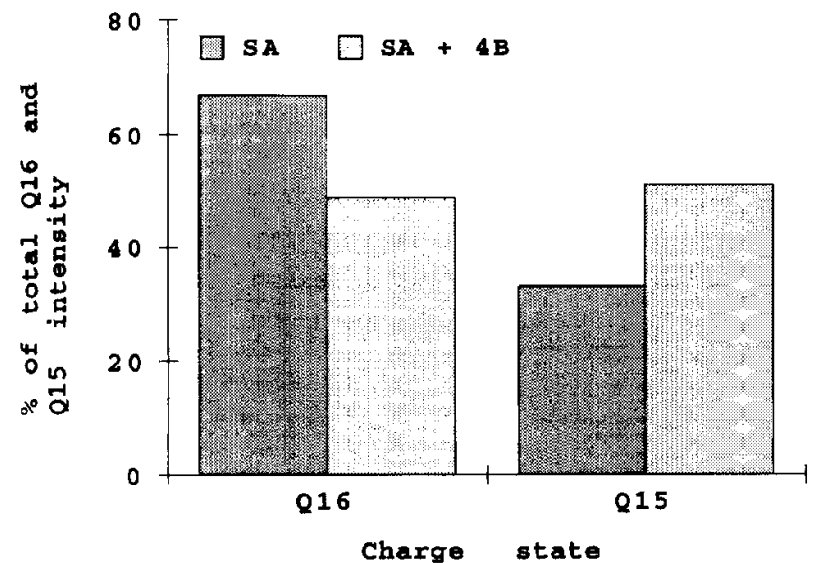

Figure 4. Bar graph representation of the intensity change of Q15 and Q16 from Figure 3a and e. The sum of the Q15 and Q16 intensities of $\mathrm{SA}$ and $\mathrm{SA}+4 \mathrm{~B}$ is normalized to $100 \%$. The bars represent the areas of the signals.

streptavidin tetramer and the four biotinylation products obtained with $40-$ and $60-\mu \mathrm{M}$ biotin are listed in Table 1 . The molecular masses for the partially separated tetrameric streptavidin that carries zero to three molecules of biotin did not deviate significantly from their theoretical mass values. There was only a minor shift to higher mass values, which may be caused by the partial overlaps of the different species.

The shift in the molecular mass of the fully biotinylated tetrameric streptavidin noncovalent complex must be due to unresolved clustering with solvent molecules as indicated by the increased peak width. This effect was validated from the mass measurement of the tetrameric streptavidin molecular mass without biotin addition [calculated $M_{\mathrm{r}}=51,884$; measured $M_{\mathrm{r}}$ $=51,890 \pm 8(n=11)$; deviation $+120 \mathrm{ppm}]$. These measurements provided strong evidence that the observed signals were due to stepwise biotinylation of streptavidin. Nevertheless, it became obvious that a reduced declustering efficiency needed for the observation of undamaged protein ligand complexes caused some increased mass measurement errors.

\section{Biotin Effects on Streptavidin}

The biotin binding to avidin and its effect on the average charge state in the ESI mass spectrum already have been reported [21]. These experiments were limited by the low instrumental resolution of the low frequency extended mass range quadrupole mass spectrometer employed. With a sector field mass spectrometer and selection of nonglycosylated streptavidin it was possible to identify different biotinylation products and separate them from the tetrameric streptavidin noncovalent complex. In addition to the expected shift in the average charge state of the streptavidin tetramer, some other important characteristics of this binding process were observed. When streptavidin is 
Table 1. Mass measurements of the streptavidin tetramer and different numbers of attached biotin molecules

\begin{tabular}{lllc}
\hline Adduct & Mass measured & Deviation & Mass calc. \\
\hline \hline Streptavidin without biotin & $51,901 \pm 7(n=7)$ & $+330 \mathrm{ppm}$ & 51,884 \\
Streptavidin plus one biotin & $52,144 \pm 8(n=7)$ & $+310 \mathrm{ppm}$ & 52,128 \\
Streptavidin plus two biotin & $52,384+6(n=8)$ & $+210 \mathrm{ppm}$ & 52,373 \\
Streptavidin plus three biotin & $52,630 \pm 12(n=8)$ & $+250 \mathrm{ppm}$ & 52,617 \\
Streptavidin plus four biotin & $52,933 \pm 17(n=8)$ & $+780 \mathrm{ppm}$ & 52,861 \\
\hline
\end{tabular}

present in excess, the differently biotinylated complexes that exhibit different intensities (Figure $3 b-d$ ) may represent the intermediates produced during the saturation process of streptavidin with biotin. Especially at half saturation with biotin (Figure 3c), it appears that the fully biotinylated streptavidin is not favored over other partially biotinylated tetrameric streptavidin complexes. On this basis it is probable that biotin binding did not follow cooperative effects.

The biotinylation-induced shift in the charge state pattern of streptavidin also was influenced by the cone voltage offset when peak heights were taken as a measure. In this case the charge state pattern of the differently charged species could be distorted by the different degree of fragmentation for the different charge states as previously described. These observations demonstrated that interpretation of the shift in the charge state pattern as a result of a conformational change must be validated carefully. It can therefore be of great importance to determine possible decomposi tions by a separation of the complex and the intermediates.

\section{Dissociation of the Noncovalent Complexes}

Declustering of electrosprayed ions was facilitated by low energy $\mathrm{CAD}$ in the sampling cone-skimmer region. The efficiency of this process depended on the sampling cone voltage offset as shown for the streptavidin tetrameric complex (Figure $2 a$ and $b$ ). In addition, the high mass tailing, which indicates the degree of clustering with solvent molecules, was reduced at the same sampling cone voltage offset for Q16 in comparison to Q15 (Figure 3a). This difference was explained by the higher translational energy of the Q16 tetrameric complex compared to the equivalent Q15 species. The opposite behavior was observed for the loss of biotin from tetrameric streptavidin. Furthermore, the charge state dependence for biotin loss from tetrameric streptavidin also was observed at higher sampling cone voltage offsets (Figure $5 b$ ). Of the different charge states, the lower charge state lost biotin more easily compared with the higher charge state. With a sampling cone voltage offset of $40 \mathrm{~V}$, no significant biotin loss from biotinylated Q16 was found. However, such loss was observed for biotinylated Q15, and a reduction of the cone voltage offset to $20 \mathrm{~V}$ was necessary to prevent dissociation of biotinylated Q15 (data not shown). In the case of a collision energy effect, the higher charge state should dissociate more readily than the lower charge state. This is indeed not the case, and therefore it was assumed that the stability of the biotin streptavidin noncovalent complex was altered by the different number of protons. Charge stripping reactions, which also could be responsible for the above-mentioned effect, are unlikely to occur to a significant extent under conditions without streptavidin subunit dissociation and only minor losses of biotin. These data strongly suggest large differences in the gas phase stability of the biotin streptavidin noncovalent complex at different charge states. The "hydrophobic box" of the biotin binding site in streptavidin [25] is not likely to be affected by charges on the noncovalent complex, but the five hydrogen bridges between biotin and the tetrameric streptavidin noncovalent complex [25] may be influenced by the different charge states of the desolvated noncovalent complex in the gas phase.

As mentioned in the foregoing text, an increase of the sampling cone voltage induced dissociations of the streptavidin tetrameric complex. Interestingly, no biotinylated monomer, dimer, or trimer form of streptavidin could be detected upon cone voltage fragmentation [26] of the tetrameric streptavidin biotin noncovalent complex (Figure 5). Because each streptavidin subunit carries a binding site for biotin [25], dissociation to biotinylated streptavidin oligomers could occur without prior biotin dissociation. From the observation that biotinylated oligomers did not appear, it was concluded that in the gas phase the dissociation of the tetrameric subunit complex required significantly higher dctivation energy than the dissociation of the subunit ligand complex.

The nonbiotinylated tetrameric streptavidin was produced preferentially upon CAD rather than the partially biotinylated species when the collision energy was raised by an increased sampling cone voltage or an increased charge (Figure 5b). This effect became more pronounced at higher sampling cone voltages (data not shown). These data already pointed to the significant difference between activation energies for the dissociation of complexes that consist of streptavidin and biotin and of streptavidin alome. 


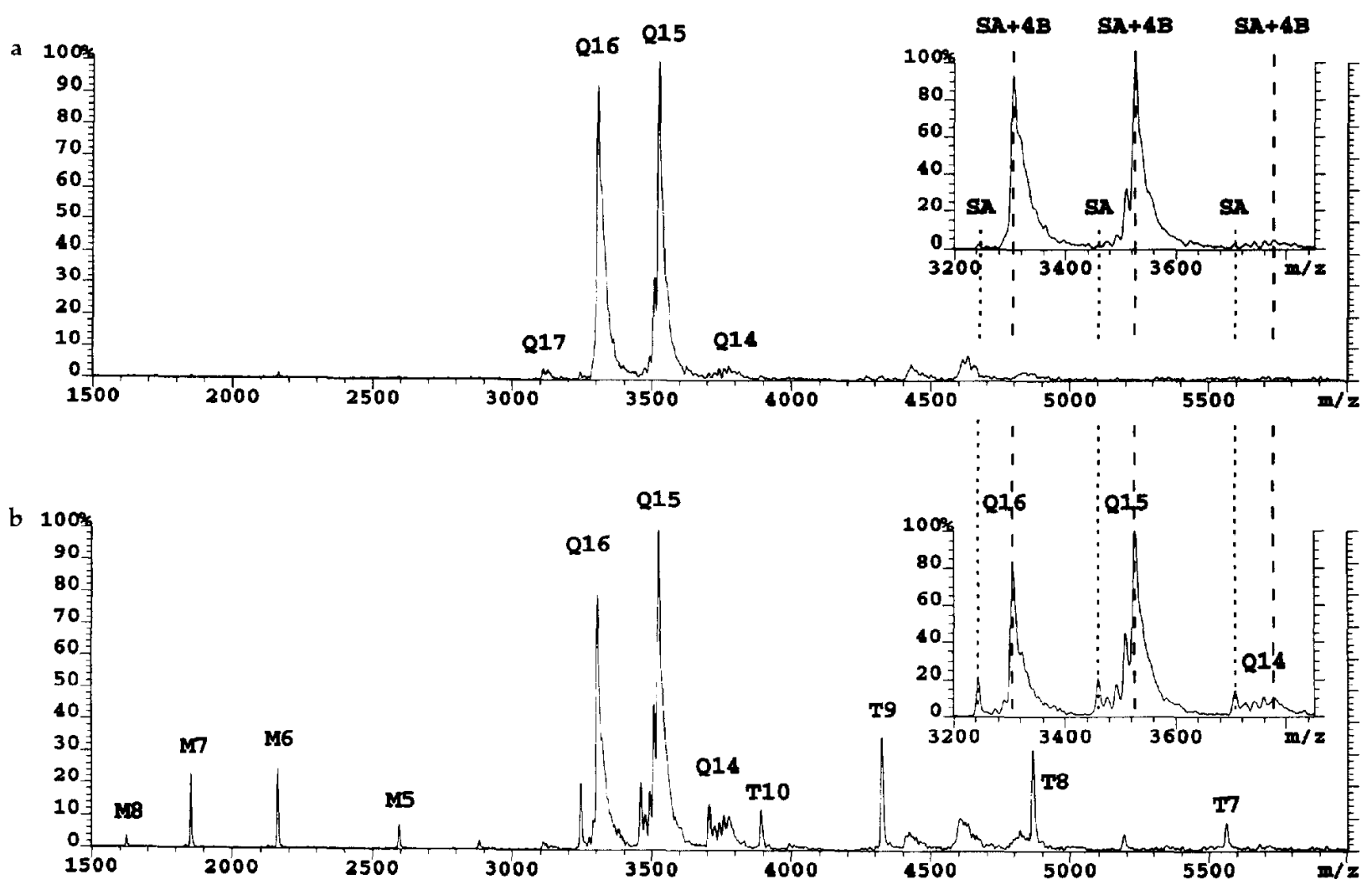

Figure 5. Partial ESI mass spectra of $1-\mu \mathrm{g} / \mu \mathrm{L}$ streptavidin in $10-\mathrm{mM}$ ammonium acetate buffer, $\mathrm{pH} \mathrm{8}$, with 80- $\mu \mathrm{M}$ biotin recorded at cone voltage offsets of (a) $40 \mathrm{~V}$ and (b) $90 \mathrm{~V}$. The insets show the expanded mass region of the tetramer. Labels: $M$, monomer; $T$, trimer; $Q$, tetramer; the number gives the number of protons; SA, streptavidin; 4B, four molecules biotin.

\section{Influence of the $k_{D}$ Value}

For the ligand binding properties the $k_{\mathrm{D}}$ value is probably of great importance. A $k_{D}$ value much smaller than the protein concentration enables binding at a concentration level of the ligand in the range of the protein concentration. In the case of a $k_{D}$ value above the protein concentration one would need ligand concentrations significantly above the protein concentration for complete binding. Therefore, the results of the binding studies are directly associated with the dimension of the $k_{\mathrm{D}}$ value. Evaluation of equilibrium data can be used in principle for determination of $k_{\mathrm{D}}$ values, but in most cases biochemical kinetic procedures may provide more information.

Conformational changes induced by glucose were expected on the basis of earlier enzymological experiments $[1,2,27,28]$. We found significant effects of glucose to hexokinase under similar electrospray conditions (Eckart, K.; Spiess, J., manuscript in preparation). These results clearly showed that the relatively small affinity $\left(k_{D}=10^{-4} \mathrm{M}\right)$ of glucose to yeast hexokinase is not too small for the survival of the enzyme substrate complex in the electrospray process. Therefore, this approach may be used further for the characterization of a variety of specific protein ligand noncovalent interactions.

\section{General Considerations}

With respect to different electrospray interface designs it should be mentioned again that the observed dissociation effects were due to sampling cone-skimmer CAD and not due to the design of the ion transport system into the mass spectrometer. Therefore, such investigations should be possible with interfaces that permit unheated electrospraying. As to the type of mass spectrometer, there is no limitation in principle for other types of mass analyzers such as quadrupole or time-of-flight (TOF) devices. The quadrupole instruments in contrast to the sector mass spectrometers may be less suited for these investigations because they are limited either in mass range or resolution, but the advanlage of such instruments is the significantly improved transmission of the interface because of the lower vacuum requirements. The development of an orthogonal ESI-TOF mass spectrometer appears to be a powerful instrument for such investigations because it combines to a certain extent the resolution capabilities and the transmission of a sector mass spectrometer with an unlimited mass range and provides detection of all ions in a time array [9, 29].

Some other aspects of this technique should be discussed also. Application of ESIMS is limited by the buffering system, which must contain volatile compo- 
nents at a concentration $\leq 100 \mathrm{mM}$. In the $100-\mathrm{mM}$ concentration range the conductivity of the ammonium acetate buffer reaches a level where changes in the mass spectral pattern were observed that were induced by the voltage applied to the spray needle. In addition to ammonium acetate we found that ammonium carbonate, ammonium bicarbonate, and triethyl ammonium acetate are compatible with the employed electrospray system. Different buffering systems created by combinations of acetic acid or formic acid as the acid component and different amines such as ammonium hydroxide or mono-, di-, or triethyl amines as the base components may be used for similar investigations. Replacement of ammonium acetate can become important when working with ammonium sensitive enzymes. In general, the sampling cone voltage offset turned out to be critical in the characterization of noncovalent protein ligand complexes and had to be optimized carefully. The temperature in the spray chamber should be kept as low as possible.

The advantage of mass spectrometry compared with NMR and $x$-ray crystallography is the relatively high sensitivity and the short time needed for the measurements. Therefore, ESIMS can be advantageous when the amount of protein for NMR analysis or for crystallization needed for x-ray crystallography is not available [2]. An interesting approach with ESIMS utilizes the direct analysis of hydrogen/deuterium $(\mathrm{H} / \mathrm{D})$ exchange rates for conformational analyses in proteins up to $20 \mathrm{ku}$ [30-33]. This technique was applied successfully to analyze the interaction between a 1-ku ligand and a 12-ku protein [34]. For larger proteins, digestion into small peptides is required for this type of analysis [35]. ESIMS analysis of $H / D$ exchange rates may be especially useful for the investigation of proteins and ligands of similar size. In such cases, it would be difficult to discriminate between different charge state patterns with regular ESIMS. However, analysis of the $\mathrm{H} / \mathrm{D}$ exchange rates by ESIMS depends on a high mass resolution and therefore cannot be applied to ligand protein systems as described here.

\section{Conclusions}

ESIMS proved to be a powerful method for the investigation of noncovalent protein complex ligand interactions. The observed significant change in the charge state pattern of the tetrameric streptavidin noncovalent complex was attributed to biotin binding induced conformational change in streptavidin. The different biotinylation states of streptavidin could be separated and analyzed with ESIMS. The separation of these different states provided insight into the biotin binding properties of streptavidin. No preference for incomplete biotinylation was observed. A larger degree of collisional-activated decompositions in the declustering region provided information on the relative strength of the noncovalent interactions in such a complex. Further attempts will be made to investigate specific ligand binding to enzymes or receptors with ESIMS.

\section{Acknowledgment}

Thomas Liepold is gratefully acknowledged for performing the Edman micro sequence analysis.

\section{References}

1. Steitz, T. A.; Harrison, R.; Weber, I. T.; Leahy, M. Ciba Found. Symp. 1983, 93, 25-46.

2. McDonald, R.; Steit<, T. A.; Engelman, D. Biochemistry 1979, $18,338-342$.

3. Katta, V.; Chait, B. T. J. Am. Chem. Soc. 1991, 113, 8534-8535.

4. Feng, R.; Konishi, Y. J. Am. Soc. Mass Spectrom. 1993, 4, $638-645$.

5. Chowdhury, S. K.; Chait, B. T. Anal. Chem. 1991, 63, $1660-1664$.

6. Ganem, B.; Li, Y.T.; Henion, J. D. J. Am. Chem. Soc. 1991, 113, $7818-7819$

7. Ganem, B.; Li, Y.-T.; Henion, J. D. I. Am. Chem. Soc. 1991, $11.3,6294-6296$.

8. Wang, G.; Cole, R. B. Anal. Chem. 1994, 66, 3702-3708.

9. Tang, X.-J.; Brewer, C. F.; Saha, S.; Chernushevich, I. V.; Ens, W.; Standing, K. G. Rapid Commun. Mass Spectrom. 1994, 8 , $750-754$

10. Siuzdak, G.; Krebs, J. F.; Benkovic, S. J; Dyson, H. J. I. Am. Chem. Soc. 1994, 116, 7937-7938.

11. Musatov, A.; Robinson, N. C. Biochemistry 1994, 33 , 10561-10567.

12. Light-Wahl, K. J.; Schwartz, B. L.; Smith, R. D. J. Am. Chem. Soc. 1994, 116, 5271-5278.

13. Haskins, N. J.; Ashcroft, A. E.; Phillips, A.; Harrison, M. Rapid Commun. Mass Spectrom. 1994, 8, 120-125.

14. Ganem, B.; Li, Y.-T.; Hsieh, Y.-L.; Henion, J. D.; Kaboord, B. F.; Frey, M. W.; Benkovic, S. J. I. Am. Chem. Soc. 1994, 116, $1352 \cdot 1358$.

15. Busman, M.; Knapp, D. R.; Schey, K. L. Rapid Commun. Mass Spectrom. 1994, 8, 211-216.

16. Winger, B. E.; Light-Wahl, K. J.; Ogorzalek Loo, R. R.; Udseth, H. R: Smith, R. D. I. Am. Soc. Mass Spectrom. 1993, 4. 536545.

17. Loo, J. A.; Ogorzalek Loo, R. R.; Andrews, P. C. Org. Mass Spectron. 1993, 28, 1640-1649.

18. Jaquinod, M.; Leize, E.; Potier, N.; Albrecht, A. M.; Shanzer, A.; van Dorsselaer, A. Tetrahedron Lett. 1993, 34, 2771-2774.

19. Huang, E. C.; Pramanik, B. N.; Tsarbopoulos, A.; Reichert, P.; Ganguly, A. K.; Trotta, P. P.; Nagabhushan, T. L.; Covey, T. R. I. Am. Soc. Mass Spectrom. 1993, 4, 624-630.

20. Smith, R. D.; Light-Wahl, K. J. Biol. Mass Spectrom. 1993, 22, $493-501$.

21. Schwartz, B. L.; Light-Wahl, K. J; Smith, R. D. J. Am. Soc. Mass Spectrom. 1994, 5, 201-204.

22. Argaraña, C. E.; Kuntz, I. D.; Birken, S.; Axel, R.; Cantor, C. R. Nucl. Acids Res. 1986, 14, 1871-1882.

23. Tijssen, P. In Practice and Theory of Enzyme Immuno Assays; Burdon, R. H.; van Knippenberg, P. H., Eds.; Elsevier: Amsterdam, 1985; p 21.

24. Pähler, A.; Hendrickson, W. A.; Gawinowicz Kolks, M. A.; Argaraña, C. E.; Cantor, C. R. I. Biol. Chem. 1987, 262, $13933 \cdots 13937$

25. Livnah, O.; Bayer, E. A.; Wilchek, M.; Sussman, J. L. Proc. Natl. Acad. Sci. USA 1993, 90, 5076-5080.

26. Loo, J. A.; Edmonds, C. G.; Smith, R. D. Anal. Chem. 1991, 63, $2488-2499$ 
27. Bennett, W. S., Jr.; Steitz, T. A. Biochemistry 1978, 75, $4848-4852$.

28. McDonald, R. C.; Engelman, D. M.; Steitz, T. A. J. Biol. Chem. 1979, 254, 2942-2943.

29. Verentchikov, A. N.; Ens, W.; Standing, K. G. Anal. Chem. 1994, 66, 126-133.

30. Stevenson, C. L.; Anderegg, R. J.; Borchardt, R. T. J. Am. Soc. Mass Spectront. 1993, 4, 646-651.
31. Anderegg, R. J.; Wagner, D. S.; Stevenson, C. L.; Borchardt, R. T. J. Am. Soc. Mass Spectrom. 1994, 5, 425-433.

32. Wagner, D. S.; Anderegg, R. J. Anal. Chem. 1994, 66, 706-711.

33. Johnson, R. S.; Walsh, K. A. Prot. Sci. 1994, 3, 2411-2418.

34. Anderegg, R. J.; Wagner, D. S. J. Am. Chem. Soc, 1995, 117, 1374-1377.

35. Ohghuro, H.; Palczewski, K.; Walsh, K. A.; Johnson, R. S. Prot. Sci. 1994, 3, 2428-2434. 